\title{
Research on the Problems and Countermeasures of Data Management in CRM System of Financial Shared Service Center
}

\author{
Ting Deng ${ }^{1, *}$

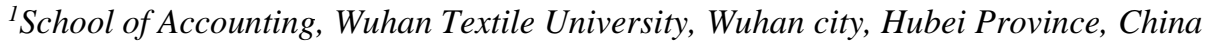 \\ *Email:Dt18171521301@163.com
}

\begin{abstract}
On the current development status of customer relationship management system of financial sharing center in China, this paper explores some problems in the application of customer data management, and puts forward some appropriate suggestions, hoping to help the customer relationship management system play a greater role in enterprises, realize the integration of industry and finance, and bring greater benefits to enterprises.
\end{abstract}

Keywords: Financial sharing, Customer relationship management system, Customer data management.

\section{INTRODUCTION}

In recent years, An accounting and reporting business management approach has emerged and prevailed -Financial Shared Service Center. It is the latest application of enterprise centralized management mode in financial management. Its purpose is to solve the problems of repeated investment and low efficiency in the construction of financial functions of large group companies through an effective operation mode. To build a financial shared service center system, we must also complete the docking of financial shared service system and other systems, such as customer management system is one of the very important modules. However, the products of domestic customer relationship management system are in the embryonic stage of the market. Many CRM software enterprises lack effective concepts and are unable to guide enterprises to implement a comprehensive customer relationship management system.

Starting from the aspect of data management in customer relationship management system, this paper studies the relevant literature, analyzes the shortcomings in its service and data mining, and puts forward some effective suggestions for related problems.

\section{OVERVIEW OF CRM}

Customer relationship management system is a continuous process for enterprises to improve their core competitiveness, continuously strengthen communication with customers, and continuously improve products and services to meet customer needs. Its content is that enterprises use information technology (IT) technology and Internet technology to collect and analyze customer multidimensional information, help enterprises identify, develop, maintain and serve customers, improve customer satisfaction, and ultimately improve enterprise income and value. Customer relationship management focuses on communication with customers, business is customer-centric, rather than the traditional product or market-centric. In order to facilitate communication with customers, customer relationship management can provide customers with a variety of communication channels.

\section{APPLICATION OF DATA MANAGEMENT MINING IN CRM}

In the era of rapid development of the Internet, for most enterprises, keeping pace with the times towards intelligent customer management is the key to the transformation. More and more enterprises will choose to start the use of customer relationship management system to complete the business needs of enterprises, in order to ensure the effect of enterprise internal management, which can help enterprises to solve the new needs in the process of development, but also can meet the different needs of different industries and 
different enterprises, help enterprise internal management to achieve better results.

However, due to the lack of the ability to find deep-seated information in a large number of data, many enterprises for the use of these data only stay in the basic layer of browsing, retrieval, query and application layer inheritance, combination, collation and other aspects of these data cannot be converted into more useful knowledge. Therefore, how to effectively manage the rapid growth of massive data in the enterprise database to improve the utilization of data resources to the advanced stage of knowledge innovation has become an urgent problem to be solved.[1]

The emerging data mining technology in recent years undoubtedly provides support for better customer relationship management. Data mining technology has been widely used in many fields at home and abroad.The research of data mining is in the ascendant, and its development prospect has been confirmed internationally. The application of data mining technology in customer relationship management is the current research hotspot, and it is also an effective means to bring practical benefits to enterprises.[2]

Many experts and scholars such as [3] un have also made a lot of research on the application of data mining in CRM. He believes that data mining technology, as a powerful method of information acquisition, is highly feasible and can extract the key information required by managers, thus effectively guiding the strategic decision-making and business management of banks and improving market competitiveness.[4] en Jinbo, from the telecommunications market, studied the telecommunications enterprise application of data mining technology massive telecommunications enterprise customer data mining analysis, from which to find a variety of potential, valuable, regular knowledge, data mining applications in CRM more mature. Domestic enterprises are also constantly developing new CRM products, constantly making the upgrade and development of CRM, its function is more perfect and rich also promote the rapid development of domestic enterprises.

\section{EXSITING PROBLEMS}

\subsection{Failure to Truly Form a Customer-centered Corporate Culture}

Corporate culture is formed in the process of long-term development of enterprises.It is the soul of the enterprise. The core idea of CRM is to take the customer as the center, so the implementation of CRM requires enterprises to change from the previous production-centered, product-centered to customer-centered. The emergence and formation of culture is a long process, and cultural change is more difficult. Therefore, in the process of implementing CRM, it is easy to make the concept of CRM contradict with the enterprise culture, and CRM fails to integrate into the enterprise culture. And many enterprises just CRM customer relationship management as information technology, rather than management philosophy, cannot cause the attention of enterprises.

\subsection{Data Collection is not Comprehensive Enough}

Domestic financial sharing is still a control model, its concept and strategic positioning error. In the application of CRM system, only for simple collection of customer information, management of customer information, the establishment of the corresponding database, information collection is not in place. The traditional ERP process and the current process is still based on market and sales, this part of the data is very lack of financial integration. Therefore, it is necessary to establish a customer relationship management system, from control to service-oriented positioning mode.

\subsection{Inadequate Data Processing Processes}

After collecting customer data, there is no in-depth analysis and classification of customer demand information, and there is a lack of system and means for the in-depth development and management of customer relationship and consumer interest value. There are big problems in service and information data mining, and customers are not truly turned into resources and wealth of enterprises through customer relationship management.

\subsection{Lack of Relevant Personnel and System Support}

Enterprise in the system planning and implementation is only responsible for technical managers, lack of senior leadership and marketing, customer support, production and other business departments involved. CRM project managers are mostly led by technical departments, and senior managers are unable to personally care about the planning and implementation of the system.

\section{RESEARCH ON COUNTERMEASURES}

\subsection{Updating the Concept of Enterprise Culture}

The core idea of CRM is to take the customer as the center, so the implementation of CRM requires enterprises to change from the previous production-centered, product-centered to customer-centered. Attract and retain customers at the 
same time, maintain a good relationship with customers, in order to maximize the mining and coordinated use of enterprise resources, including information resources, customer resources, etc., expand the living space of enterprises, enhance the core competitiveness of enterprises. This cultural change requires changes in the personnel and resources of enterprises. Through training, the employees can understand the real value of CRM system and integrate it into the enterprise culture. At the same time, the sales, marketing, technology, production, management, finance, service and other business factors are carried out around the external resources of the enterprise based on customer resources to realize the effective allocation of customer resources and internal resources. CRM as a powerful tool to support the new corporate culture, will provide protection for the implementation and implementation of corporate culture.

The key to implement CRM is that it not only tells enterprises how to serve customers, more importantly, it can provide customers with more personalized services, so that the customer' $\mathrm{s}$ life is simpler and more comfortable.

\subsection{Collect and Classify Customer Data}

\subsubsection{Customer Data Collection}

The main goal of customer data collection in this stage is to ensure the comprehensiveness, accuracy and timeliness of customer data. What data should be collected from customers are shown in Figure 1.

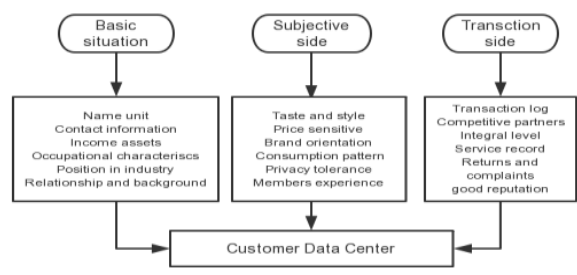

Figure1 Customer data center

For a mature database system, the source of information data is required to be stable and reliable. It is necessary to establish a multi-channel integrated customer information collection platform (Figure 2).

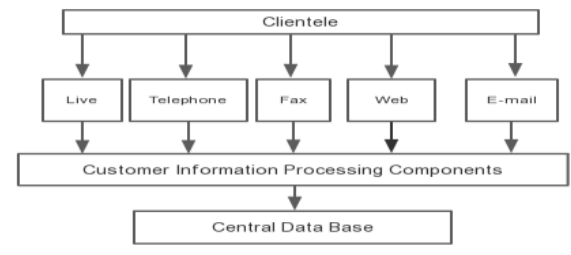

Figure 2 multi-channel integrated customer information collection platform

\subsubsection{Classification of Customer Data}

From the overall idea of CRM, it can be roughly divided into the following categories: Existing customers ; potential customers ; loss of customers; Distributors.

CRM system customer information is generally obtained from the sales process, customer service process, business promotion process or other forms. From the perspective of the needs of business activities, there is a process of understanding customers, targeted promotion and transaction. The behavior process generates different data types, and thus customer data are divided into three categories.

\subsubsection{Customer Descriptive Data}

Such data are data types that describe customers or consumers. They are usually tabular summary data. In terms of relational database, they are different columns in a customer data. Because is the customer' $\mathrm{s}$ basic information, change is not fast, can be used for a longer period of time.

\subsubsection{Market Promotion Data}

The detailed design of promotional activities for each customer depends on the complexity of the CRM database system.

\subsubsection{Customer Transaction Data}

All data describing the interaction between enterprises and customers belong to customer transaction data. Such data and promotional activity data will change rapidly over time. Therefore, it requires that this storage structure can easily support the update and change of transaction data with time tags.

The purpose of classification is to allow managers to ' bird 's eye view ' the data in the whole database from a higher level, so that they can use different standards or methods to treat customers in different subdivision groups, and provide targeted and more personalized services.

\subsection{Improving Data Processing Flow}

Customers are the basis of enterprise competition, and customer data are the core soul of CRM system. The processing and analysis of data are the main tasks and functions of CRM.[5] hen Bin and Jiang Shaozhong two scholars in the ' customer relationship management system ( CRM ) data processing ' article on the data processing process. All our data are divided into two parts: the front data and the back data. Front zone data refers to data from outside customers. The characteristics of such data are mainly massive, discrete, unstructured and to be verified. Back zone data is the 
information flow from the operation process of the enterprise itself, such as parts, semi-finished products, inventory of finished products, the current production capacity, after-sales service ability and sales channel data and so on. Such data can be integrated through the internal network of the enterprise and the database of the relevant departments to form information for decision-making in different departments. Since it comes from within the enterprise, such data are easy to obtain, credible, concentrated and limited for a mature enterprise.

After collecting customer information data as comprehensively as possible through multiple channels, the quality of data processing process also directly determines whether the CRM system can successfully run in the enterprise and gain competitive advantage for the enterprise. The following figure shows the logic of data processing in CRM system(Figure 3) :

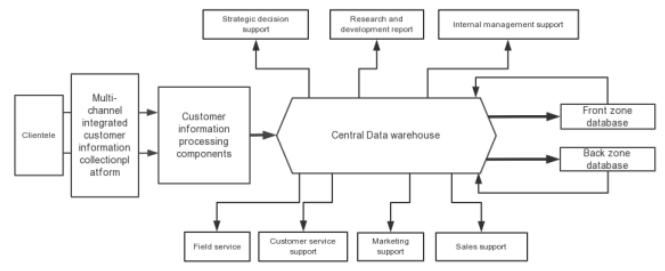

Figure3 data processing flow

CRM system data processing generally includes the above five aspects: multi-channel customer information collection platform, customer information processing components, front area database, back area database, central database.

\subsubsection{Multi-channel Customer Information Collection Platform.}

This platform connects enterprises and customers closely and undertakes the interface responsibilities. Enterprises provide relevant service support by understanding customer needs and receiving product feedback information. It can also publish product information to customers or potential customers and accept customer complaints.

The use of multi-channel customer information collection platform needs to follow three principles. The first and most important point is 'customer-centred '. The needs of enterprise management are based on customer needs rather than some requirements of the enterprise itself. Whether this platform can really work depends on whether it can be accepted by customers and form a two-way interaction with enterprises through it. The second principle is enterprise-oriented. Enterprises should choose information integration channels according to their own development, such as the characteristics of products or services, the characteristics of customer groups, and their own technology and capital strength. The third principle is the principle of combining with corporate strategy. CRM projects must be combined with the long-term strategy of enterprises, and learn to stand on the current and look at the future.

\subsubsection{Customer Information Processing Components}

Since the data collected from the target customer group are generally discrete, unstructured and to be verified, there are many invalid or even misleading information. This requires the use of scientific methods to clean, refine these massive data, to achieve the purpose of rough refinement, so as to provide business, decision-making support for departments at all levels of the enterprise.Generally speaking, customer information processing has three steps :

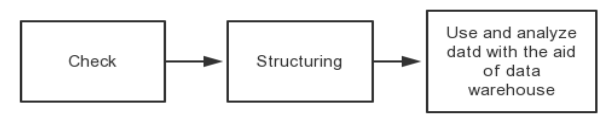

In order to provide better services to customers and improve customer satisfaction, enterprises need to adopt scientific processing methods to intercept data and analyze data, but in fact there are still a lot of information is nonlinear, unstructured, computer processing such information often powerless. This requires the company 's purchasing behavior analysis experts, consumer behavior analysis experts to deal with.

\subsubsection{Central Data Warehouse and Front and Back Database}

Customer database is the core of using and mining customer information, and its establishment is the basis of all data analysis. With the wide application and development of computer technology, people are no longer satisfied with simply performing data transaction operations, but require systematic organization, understanding, analysis and reasoning of existing data, so as to quickly and accurately obtain associated information and provide basis for strategic decision-making. Data warehouse is a technical solution to the above problems. It is the core of decision support system environment based on large-scale database. In the CRM data processing process, the central data warehouse is the most important. The front and back area database is a data mart that flows into different departments of the company from the data warehouse to support the customized use of these departments. The relationship between front and back zone database and central data warehouse is the relationship between subordinate and subordinate. In the construction of enterprise's CRM, there is no universal model suitable 
for different types of enterprises, which requires enterprises to proceed from the specific situation and keep pace with the times. Continuous analysis of data processing problems, find suitable enterprise development data warehouse, so as to promote enterprise development.

\subsection{Strengthening Personnel Training and System Support}

Enterprises should implement the corresponding training for the CRM scheme. The training objects mainly include sales personnel, service personnel and management personnel. The purpose of training is to enable the users of the system to master the use method and understand the management and maintenance needs after the implementation of the scheme, so that the CRM system can be successfully operated. In addition, enterprises need to integrate data warehouse, data mining and other technologies, and linked with e-commerce, but also with ERP, SCM integration, better promote the operation of enterprise CRM system.

\section{CONCLUSION}

In general, the development of CRM system in China is still in the stage of continuous improvement and maturity. For enterprises, the active implementation of CRM customer relationship management system has become increasingly important. Rational use of CRM system can create more opportunities and infinite possibilities for enterprises, so that enterprises can better be based on the complex market environment. The key to the success of CRM system is data processing. How to build CRM system data processing process has no fixed mode, need the enterprise according to their own situation reasonable planning. As Yang Jian, founder of Teamface, said: 'CRM customer relationship management import is not overnight things, companies need to constantly explore, develop, keep pace with the times.'

\section{REFERENCES}

[1] Li Xianglin; Jin Zhonghui; Liu Dan. Analysis of data mining process in customer relationship management $[\mathrm{J}]$. Business report, 2012, (07): $277-278$

[2] Sun Ning. Research on customer relationship management system based on data mining [J]. Technology and industry, 2011 (06) : $59-61$.

[3] Cao Jun. Research on the application of data mining technology in bank customer relationship management [C]. Hunan University, 2013.

[4] Chen Jinbo. Data mining application research for telecom CRM [C]. Southeast University, 2006.

[5] Chen Bin ; Jiang Shaozhong.Data processing in customer relationship management system ( CRM ) [J] enterprise economy, 2002 (11). 